\title{
Centrality Dependence of Hadron Multiplicities in Nuclear Collisions in the Dual Parton Model
}

\author{
A. Capella and D. Sousa \\ Laboratoire de Physique Théorique円 \\ Université de Paris-Sud, Bâtiment 210, F-91405 Orsay Cedex, France
}

\begin{abstract}
We show that, even in purely soft processes, the hadronic multiplicity in nucleusnucleus interactions contains a term that scales with the number of binary collisions. In the absence of shadowing corrections, this term dominates at mid rapidities and high energies. Shadowing corrections are calculated as a function of impact parameter and the centrality dependence of mid-rapidity multiplicities is determined. The multiplicity per participant increases with centrality with a rate that increases between SPS and RHIC energies, in agreement with experiment.
\end{abstract}

LPT Orsay 00-137

December 2000

\footnotetext{
${ }^{1}$ Unité Mixte de Recherche - CNRS - UMR N 8627
} 


\section{Introduction}

Hadron multiplicities per unit rapidity at SPS energy show an approximate scaling with the number of participants. This property is known as "wounded nucleon model" (WNM) [1]. However, a precise determination of the ratio $d N^{c h} / d \eta\left(\eta^{*}=0\right) / n_{\text {part }}$, both at CERN-SPS [2] and at RHIC [3], shows a steady increase from peripheral to central collisions. This increase is larger at RHIC energies, where the data show no saturation for the most central bins.

It is a widespread belief that "soft" processes lead to a scaling in $n_{\text {part }}$ while "hard" ones lead to a scaling with the number $n$ of binary collisions. While, this is the case for total cross-sections, it is not so for single particle inclusive cross-sections. If one neglects the effects of shadowing (i.e. nuclear effects in structure functions associated to triple Pomeron interactions), there is a theorem known as Abramovski, Gribov, Kancheli (AGK) cancellation [4], based on general principles, according to which $d \sigma^{c h} / d y$ scales with $n$ at mid-rapidities and asymptotic energies. The best way to understand this cancellation is to illustrate it with a model that satisfies these general principles, namely the Glauber model.

Let us consider for simplicity $p A$ scattering. The cross-section for $n$ inelastic collisions of the proton with $n$ nucleons of the target is given by the probabilistic expression

$$
\sigma_{n}^{p A}(b)=\left(\begin{array}{l}
A \\
n
\end{array}\right)\left(\sigma_{p p} T_{A}(b)\right)^{n}\left(1-\sigma_{p p} T_{A}(b)\right)^{A-n}
$$

where $T_{A}(b)$ are nuclear profile functions normalized to unity. Using this equation it is easy to see that

$$
\sigma_{i n}^{p A}=\int d^{2} b \sum_{n=1}^{A} \sigma_{n}^{p A}(b) \propto A^{2 / 3} .
$$

However, for the non-diffractive inclusive cross-section one has

$$
\frac{d \sigma^{p A}}{d y} \propto \int d^{2} b \sum_{n=1}^{A} n \sigma_{n}^{p A}(b) \propto A^{1} .
$$

Thus, one obtains the behaviour $A^{1}$ typical of hard processes.

The derivation of (3) assumes that the inclusive cross-section for $n$ inelastic collisions is $n$ times the corresponding one for a single collision (or, more generally, that the hadronic plateau produced in an inelastic collision does not depend on the number of inelastic collisions). Clearly, such a condition is only true at mid-rapidities and for 
asymptotic energies. A basic idea in the WNM is that a nucleon (here the projectile), when wounded once, looses its ability to produce extra particles in further collisions. This may be valid at low energies, when the projectile undergoes successive collisions with the nucleons of the target. However, at high energies, the space-time development of the interaction implies that the $n$ collisions are "parallel", i.e. they originate from different constituents of the projectile wave function and take place simultaneously. (Technically, it means that one is dealing with non-planar diagrams). This is the so-called GlauberGribov model [5]. A more detailed discussion can be found in [6].

As discussed above, at finite energies the constraints of energy-momentum conservation lead to a violation of the AGK cancellation. The dual parton model (DPM) [7] and the quark gluon string model (QGSM) [8], while obeying AGK cancellation at mid rapidities and asymptotic energies, contain an "educated guess" on its violation at finite energies. These models are based on the quark-gluon content of hadrons in the framework of the Glauber-Gribov model, and on the large $N$ expansion of non-perturbative QCD. The charged multiplicities per unit rapidity are given by [7, 9]

$$
\begin{aligned}
& \frac{d N_{A A}^{c h}}{d y}(y, b)=n_{A}(b)\left[N_{\mu}^{q q^{P}-q_{v}^{T}}(y)+N_{\mu}^{q_{v}^{P}-q q^{T}}(y)+(2 k-2) N_{\mu}^{q_{s}-\bar{q}_{s}}\right]+ \\
& \left(n(b)-n_{A}(b)\right)\left(2 k N_{\mu}^{q_{s}-\bar{q}_{s}}(y)\right) .
\end{aligned}
$$

Here $P$ and $T$ stand for the projectile and target nuclei,

$$
n(b)=\sigma_{p p} \int d^{2} s A^{2} T_{A}(s) T_{A}(b-s) / \sigma_{A A}(b) \equiv \sigma_{p p} A^{2} T_{A A}(b) / \sigma_{A A}(b)
$$

is the average number of binary collisions and

$$
n_{A}(b) \equiv n_{\text {part }}(b) / 2=\int d^{2} s A T_{A}(s)\left[1-\exp \left(-\sigma_{p p} A T_{A}(b-s)\right] / \sigma_{A A}(b)\right.
$$

the average number of participants of nucleus $A . k$ is the average number of inelastic collisions in $p p$ and $\mu(b)=k n(b) / n_{A}(b)$ is the average total number of collisions suffered by each nucleon. The first term in (4) is the plateau height in a $p p$ collision, resulting from the superposition of $2 k$ strings, multiplied by $n_{A}$. Since in DPM there are two strings per inelastic collision, the second term, consisting of strings stretched between sea quarks and antiquarks, makes up a total number of strings equal to $2 \mathrm{kn}$. 
The hadronic multiplicities of the strings in (4) are obtained from a convolution of momentum distribution function and fragmentation functions (eqs. (3.1) to (3.4) of [7]). The former are given [7] by a product of Regge propagators times a delta function of energy conservation:

$$
\rho_{k n}\left(x_{1}, x_{2 k n} ; x_{2}, x_{3}, \ldots, x_{2 k n-1}\right)=c_{k n} x_{1}^{-1 / 2} x_{2 k n}^{3 / 2} x_{2}^{-1} \ldots x_{2 k n-1}^{-1} \delta\left(1-\sum_{i=1}^{2 k n} x_{i}\right)
$$

Here $x_{1}$ and $x_{2 k n}$ denote the $x$-values of the valence quark and diquark, respectively, $x_{2} \ldots x_{2 k n-1}$ those of the sea quarks and antiquarks and $c_{k n}$ is obtained by normalizing $\rho_{k n}$ to unity. The momentum distribution of a single constituent is obtained by integrating (7) over the $x_{i}$ 's of all others. In order to regularize the singularities at $x_{i} \sim 0$ all $x_{i}(i \neq 2 k n)$ in eq. (7) are replaced by [10] $\bar{x}_{i}=\left(x_{i}^{2}+4 \mu^{2} / s\right)^{1 / 2}$ with $\mu^{2}=0.1 \mathrm{GeV}^{2}$. This introduces a free parameter $\mu$ in the model. The diquarks and quark fragmentation functions into charged particles were determined from $e^{+} e^{-}$and/or $p p$ data. They are given by [10]

$$
\begin{aligned}
z D_{q q}(z) & =1.12(1-z)^{3} \\
z D_{q_{v}}=z D_{q_{s}}(z) & =\frac{1.12}{1.35} \frac{1.3(1-z)^{2}+0.05}{1-0.5 z}
\end{aligned}
$$

Finally, we have taken for the threshold of $q q-q$ strings $s_{0}=\left(m_{p}+m_{\pi}^{T}\right)^{2}$ and for the one of $q-\bar{q}$ strings $s_{0}=\left(2 m_{\pi}^{T}\right)^{2}$, with $m_{\pi}^{T}=0.33 \mathrm{GeV}$. The value of $k$ can be determined in a generalized eikonal model. Alternatively, we can fix it in such a way that the plateau in $p p$ agrees with the experimental value of $d N^{c h} / d y\left(y^{*}=0\right)$ for non-diffractive events. These two determinations are consistent with each other [7, 8].

Note that at asymptotic energies, when the plateau height of all strings coincides (i.e. $N^{q q-q_{v}}(y=0) \simeq N^{q-\bar{q}_{s}}\left(y^{*}=0\right)$ ) one recovers the AGK cancellation, namely

$$
\begin{aligned}
& \frac{d N_{A A}^{c h}}{d y}\left(b, y^{*}=0\right) \sim n(b) 2 k N^{q_{s}-\bar{q}_{s}}\left(y^{*}=0\right) \\
& \sim \frac{A^{2} T_{A A}(b)}{\sigma_{A A}(b)} \sigma_{p p}^{N D} \frac{d N_{p p}^{N D}}{d y}\left(y^{*}=0\right)=\frac{A^{2} T_{A A}(b)}{\sigma_{A A}(b)} \frac{d \sigma_{p p}^{N D}}{d y}\left(y^{*}=0\right) .
\end{aligned}
$$

Already at RHIC energies, where $N^{q_{s}-\bar{q}_{s}}\left(y^{*} \sim 0\right)$ is substantially smaller than $N^{q q-q}\left(y^{*} \sim\right.$ 0 ), the last term of (4) turns out to dominate. However, energy conservation constraints produce a decrease of $N_{\mu}^{q_{s}-\bar{q}_{s}}\left(y^{*}=0\right)$ with increasing centrality and, thus, the scaling in the number of binary collisions is not reached. Since the $p p$ rapidity plateau $d N_{p p}^{N D} / d y=$ 
$\left(1 / \sigma_{p p}^{N D}\right) d \sigma_{p p}^{N D} / d y$ refers to non-diffractive events, for consistency we have to use also the non-diffractive cross-section $\sigma_{p p}^{N D}$ (rather than the inelastic one) in the determination of $n_{A}$ and $n$, in order to recover eq. (9) asymptotically?.

We can now compute the centrality dependence of hadronic multiplicities. We present first the results obtained in the absence of shadowing, at three different energies : $\sqrt{s}=17.3,130$ and $200 \mathrm{GeV}$. The corresponding non-diffractive cross-sections are [11, 12] $\sigma_{N D}=26,33$ and $34 \mathrm{mb}$, respectively. We take $k=1.4,2.0$ and 2.2, corresponding to $d N_{p p}^{N D} / d y=1.56,2.72$ and 3.04 [11, 13]. The values of the charged multiplicities $N_{\mu}^{q q-q}\left(y^{*}=0\right)$ and $N_{\mu}^{q-\bar{q}}\left(y^{*}=0\right)$ of the individual strings calculated in DPM, are listed in Table 1. The results are shown in Fig. 1 (solid line) and in Figs. 2-3 (dashed lines). At SPS we obtain a mild increase of the multiplicity per participant consistent? with the results of the WA98 collaboration [2] (see Fig. 1). This increase gets stronger with increasing energies (Figs. 2 and 3).

It should be noted that the absolute value of the multiplicity at SPS energies determined in DPM has some uncertainty. First, the excess of $K^{+}$and $p$ over $K^{-}$and $\bar{p}$ is not properly taken into account in the above calculation. Second, the value of the multiplicity in the $q_{s}-\bar{q}_{s}$ strings is affected by the value of its threshold. The latter has much less effect at $\sqrt{s}=130$ and $200 \mathrm{GeV}$. However, shadowing corrections, which are negligibly small at SPS energies, become important at RHIC and have to be taken into account. This introduces some uncertainty at RHIC energies (see below).

As emphasized in [15], shadowing corrections in Gribov theory are universal, i.e. they apply both to soft and hard processes. They are closely related to the size of diffractive production and, thus, are controlled by triple Pomeron diagrams [15, 16]. In the recent papers [17] it was shown, that, when unitarity corrections are consistently taken into account, one can describe hard diffraction at HERA and soft one (photoproduction) with the same value of the triple Pomeron coupling. This value agrees with the one in ref. [16] and will be used in the following calculation. The reduction of the multiplicity resulting from shadowing corrections is given by the ratio [15]

$$
R_{A B}(b)=\frac{\int d^{2} s f_{A}(s) f_{B}(b-s)}{T_{A B}(b)}
$$

\footnotetext{
${ }^{2}$ Conventionally one uses instead $\sigma_{\text {inel }}$. In this case one should also use the $p p$ multiplicity for inelastic events. Since the latter is about $10 \%$ smaller than the non-diffractive one [11], the two effects tend to compensate with each other and only a small difference remains in the calculated multiplicities.

${ }^{3}$ The opposite claim was made in [14] based on a model which is an over-simplified version of DPM.
} 
where

$$
f_{A}(b)=\frac{T_{A}(b)}{1+A F(s) T_{A}(b)} .
$$

Here the function $\mathrm{F}$ is given by the integral of the ratio of the triple Pomeron cross-section $d^{2} \sigma^{P P P} / d y d t$ at $t=0$ over the single Pomeron exchange cross-section $\sigma_{p}(s)$ :

$$
F(s)=\left.4 \pi \int_{y_{\min }}^{y_{\max }} d y \frac{1}{\sigma_{P}(s)} \frac{d^{2} \sigma^{P P P}}{d y d t}\right|_{t=0}=C\left[\exp \left(\Delta y_{\max }\right)-\exp \left(\Delta y_{\min }\right)\right]
$$

with $y=\ln \left(s / M^{2}\right)$, where $M^{2}$ is the squared mass of the diffractive system. For a particle produced at $y_{c m}=0, y_{\max }=\frac{1}{2} \ell n\left(\frac{s}{m_{N}^{2}}\right)$ and $y_{\min }=\ell n\left(R_{A} m_{N} / \sqrt{3}\right)$. Using the parameters of [14] [16] we get : $C=0.31 \mathrm{fm}^{2}$.

The values of the shadowing corrections at each impact parameter $R(b)$ in $A u-A u$ collisions at $\sqrt{s}=130 \mathrm{GeV}$ are shown in Table 1. Our results including shadowing are shown by the upper lines of the dark bands in Figs. 2 and 3.

It should be stressed that our calculations refer to $d N / d y$ while the first RHIC measurements [3] [18] refer to $d N / d \eta$. The latter is, of course, smaller at mid rapidities. This difference is negligibly small as SPS where the laboratory pseudo-rapidity variable is used. However, at $\sqrt{s}=130$ and $200 \mathrm{GeV}$, where $\eta_{c m}$ is used instead, their ratio is as large as 1.3 [19]. This value is substantially larger than the value of 1.1 quoted in [3].

Our results for the centrality dependence of $d N / d \eta$ per participant at $\sqrt{s}=$ $130 \mathrm{GeV}$ are shown in Fig. 4 (upper line of the dark band) and compared with the PHENIX data. As we see, the centrality dependence is quite well reproduced. However, the absolute values are about $15 \%$ higher than the data.

It should be stressed that the values of $R$, eq. (10) are quite large (see Table 2). As pointed out in [13] they have a rather large uncertainty at RHIC energies. An alternative calculation of $R$ in ref. [13], based on a formalism that reproduces the nuclear effects in DIS on nuclei, led to values of shadowing about $15 \%$ larger than the ones obtained here. Clearly, with these larger values of the shadowing corrections we would obtain a quantitative agreement with the PHENIX data.

Multiplying the values of $R_{A u} A u(b)$ in Table 2 by a factor 0.85 we obtain the lower lines of the dark bands in Figs. 2-4. These bands can be regarded as an estimate of

\footnotetext{
${ }^{4}$ The increase of $\left\langle p_{T}\right\rangle$ with centrality is predicted to be rather small at RHIC [20]. Thus, we have used the same 1.3 reduction factor for all centralities.
} 
the uncertainties on the values of the calculated multiplicities due to uncertainties in the shadowing corrections. Note that these uncertainties affect mostly the absolute values of the multiplicities - while their centrality dependence is determined quite unambigously within our model.

The multiplicity per participant at SPS energies increases by 1.15 between $b=$ $10 \mathrm{fm}$ and $b=0$. The corresponding increase at $\sqrt{s}=130 \mathrm{GeV}$ and $200 \mathrm{GeV}$ is 1.30 and 1.31, respectively. As a consequence of this saturation, the rise of the central plateau in $A u$ - $A u$ collisions between these two energies is close to the one in $p p$ collisions - an interesting prediction of our model.

We would like to compare our results with the ones obtained in other approaches. Many Monte Carlos based on or inspired by DPM or QGSM do contain a term proportional to the number of binary collisions. However, in other approaches [21, 22] such a term is associated with minijets. Of course minijets are produced at high energies. They have been incorporated in DPM and modify the $p_{T}$ dependence of the model [23]. However, they do not affect the multiplicities, since they play the same role as $q_{s}-\bar{q}_{s}$ strings and the total number of such strings is controlled by unitarity. A comparison with [22] shows that, while in this approach the multiplicity is given by a linear combination of $n(b)$ and $n_{\text {part }}(b)$, with coefficients independent of $b$, in our case these coefficients decrease with increasing centrality. More important, in our approach these coefficients are calculated while in 22 they are fitted to the data at each energy.

An interesting estimate of the centrality dependence of charged multiplicities in high density QCD [24] has been presented in 22]. Surprisingly the result at $\sqrt{s}=130 \mathrm{GeV}$ is almost identical to the one based on the minijet picture obtained in [22]. However, this result relys entirely on the logarithmic dependence of the gluon structure function of the nucleon on the saturation scale $Q_{s}^{2}$, used in [22] (see last paper of ref. 24] for a discussion on this point). It is also interesting to remark that the centrality dependence of the multiplicity per participant in the minijet model [22] gets stronger with increasing energy - due to an increase of the minijet fraction. On the contrary, in high density QCD the effect is the opposite one, namely, the (partonic) multiplicity per participant depends less and less on centrality when energy increases density QCD saturation model. However, in the RHIC energy range, from $\sqrt{s}=130$ to

\footnotetext{
${ }^{5}$ This is due to the fact that the geometrical factor $\rho_{\text {part }}(b)$ in $Q_{s}^{2}$, which depends strongly on centrality, is independent of energy, while the factor $x G(x)$, which depends on impact parameter very mildly, increases with $s$. Therefore $\ln Q_{s}^{2} \propto \ln [x G(x)]$ at very high energies.
} 
$200 \mathrm{GeV}$, this effect is negligeably small and the centrality dependence of the multiplicity per participant is the same at these two energies. Interestingly, the same result is obtained in DPM when shadowing corrections are taken into account.

\section{Acknowledgments}

It is a pleasure to thank Y. Dokshitzer, A. Kaidalov, A. Krzywicki, A. Mueller, C. Pajares, C. Salgado and D. Schiff for interesting discussions. D.S. thanks Fundación Barrie de la Maza for financial support. 


\section{References}

[1] A. Bialas, A. Bleszynski and W. Czyz, Nucl. Phys. B111, 461 (1976).

[2] WA98 collaboration, M. M. Aggarwal et al, nucl-ex/0008004.

[3] PHENIX collaboration, K. Adkox et al, nucl-exp/0012008.

[4] V. A. Abramovsky, V. N. Gribov and O. V. Kancheli, Sov. J. Nucl. Phys. 18, 308 (1974).

[5] V. N. Gribov, JETP 56, 812 (1969).

V. N. Gribov, JETP 57, 1306 (1969).

[6] A. Capella and A. Krzywicki, Phys. Rev. D18, 3357 (1977).

[7] A. Capella, U. Sukhatme, C.I. Tan and J. Tran Thanh Van, Phys. Rep. 236, 225 (1994).

[8] A. B. Kaidalov, Surveys in High Energy Physics 13, 265 (1999).

[9] A. Capella, J. Kwiecinski and J. Tran Thanh Van, Phys. Lett. 108B, 347 (1982).

A. Capella, C. Pajares, A. Ramallo, Nucl. Phys. B241, 75 (1984).

A. Capella, C. Merino and J. Tran Thanh Van, Phys. Lett. B265, 415 (1991).

[10] A. Capella and J. Tran Thanh Van, Z. für Phys. 10, 249 (1981).

[11] Particle Data Group, Euro Phys. J. 15, 1 (2000).

[12] D. Bernard et al, Phys. Lett. 186B, 227 (1987).

[13] UA5 collaboration, Phys. Rep. 154, 247 (1987).

[14] J. Dias de Deus, R. Ugocioni, Phys. Lett. B491, 253 (2000) ; hep-ph 0009288.

[15] A. Capella, A. Kaidalov, J. Tran Thanh Van, Heavy Ion Physics 9 (1999).

[16] A. Capella, A. Kaidalov, C. Merino, D. Pertermann and J. Tran Thanh Van, Euro Phys. J. C5, 111 (1998).

[17] A. Capella, E. G. Ferreiro, A. Kaidalov and C. Salgado, Nucl. Phys. B593, 336 (2001) and hep-ph 0007236 (to be published in Phys. Rev. D). 
[18] PHOBOS collaboration, B. B. Back et al, Phys. Rev. Lett. 85, 3100 (2000).

[19] P. Aurenche, F. Bopp, A. Capella, J. Kwiecinski, M. Maire, J. Ranft and J. Tran Thanh Van, Phys. Rev. D45, 92 (1992).

[20] A. Capella, E. G. Ferreiro, A. Kaidalov, Eur. Phys. J C11, 163 (1999).

[21] X.-N. Wang and M. Gyulassy, nucl-th 0008014.

[22] D. Kharzeev and M. Nardi, nucl-th 0012025.

[23] A. Capella, J. Kwiecinski and J. Tran Thanh Van, Phys. Rev. Lett. 58, 2015 (1987).

[24] L. Mc Lerran and R. Venugopalan, Phys. Rev. D49, 2233 (1994).

E. Iancu, A. Leonidov and L. Mc. Lerran, hep-ph 0011241.

A. H. Mueller, Nucl. Phys. B572, 227 (2000).

E. Iancu and L. Mc Lerran, hep-ph 0103032. 


\section{Figure Captions :}

Figure 1. The values of $d N^{c h} / d y / n_{\text {part }}$ versus $n_{\text {part }}$ for $P b P b$ collisions at $\sqrt{s}=17.3 \mathrm{GeV}$ in the range $-0.5<y_{\mathrm{cm}}<0.5$ computed from eqs. (4) to (6), compared with the WA98 data [2] for $d N / d \eta / n_{\text {part }}$. The difference between $d N / d \eta$ and $d N / d y$ is very small since the laboratory pseudo-rapidity is used (see main text).

Figure 2. The values of $d N^{c h} / d y /\left(0.5 n_{\text {part }}\right)$ for $A u$ - $A u$ collisions at $\sqrt{s}=130 \mathrm{GeV}$ in the range $-0.35<y_{\mathrm{cm}}<0.35$ computed from eqs. (4) to (6) (dashed line). The upper line in the dark band, is obtained after shadowing corrections computed from eqs. (10)-(12). The lower one is obtained with a different determination of the shadowing corrections (see main text).

Figure 3. Same as Fig. 2 for $\sqrt{s}=200 \mathrm{GeV}$.

Figure 4. Same as Fig. 2 for $d N^{c h} / d \eta_{c . m}$. The dashed line (results without shadowing) has been omitted here. The PHENIX data [3] are also shown (black circles and shaded area).

\section{Table Caption :}

Values of $N_{\mu}^{q q-q}$ and $N_{\mu}^{q-\bar{q}}$ in eq. (4) at $\sqrt{s}=130 \mathrm{GeV}$ computed in DPM at different values of the impact parameter. The values correspond to charged particles per unit rapidity in the range $-0.35<y_{\mathrm{cm}}<0.35$. The corresponding shadowing corrections $R_{A u A u}(b)$ computed from eqs. (10-12) are given in the third column. 
Fig. 1

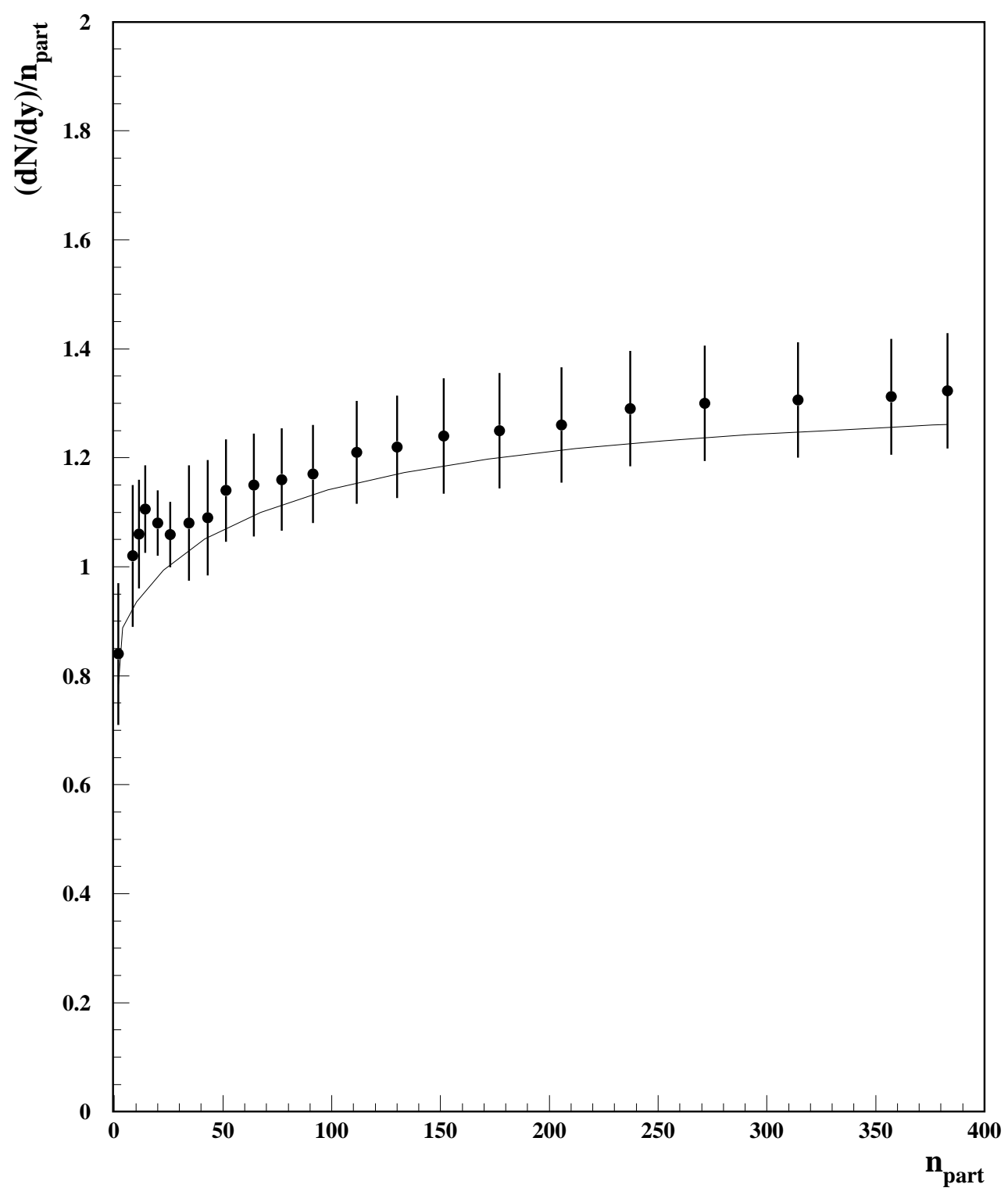


Fig. 2

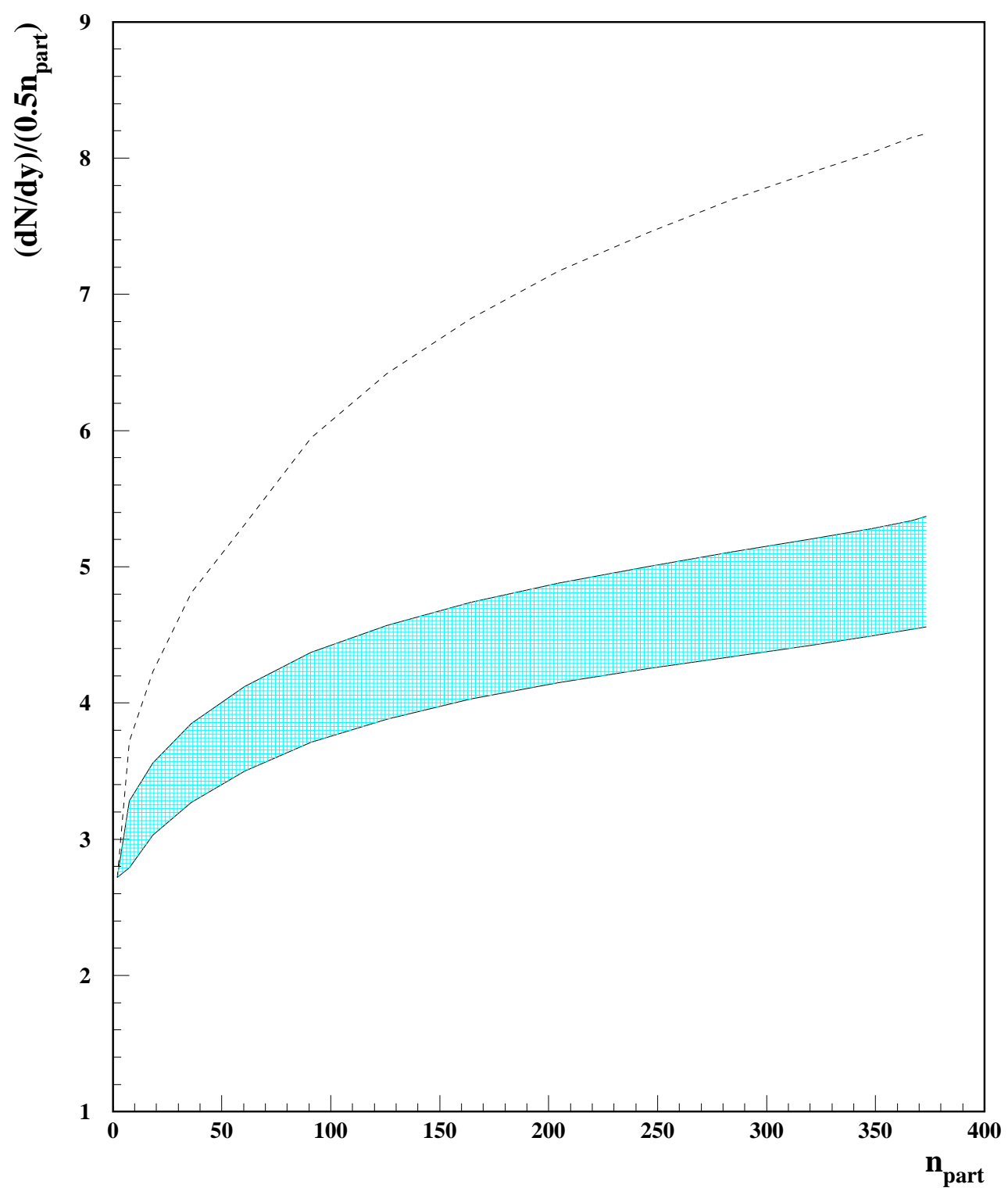


Fig. 3

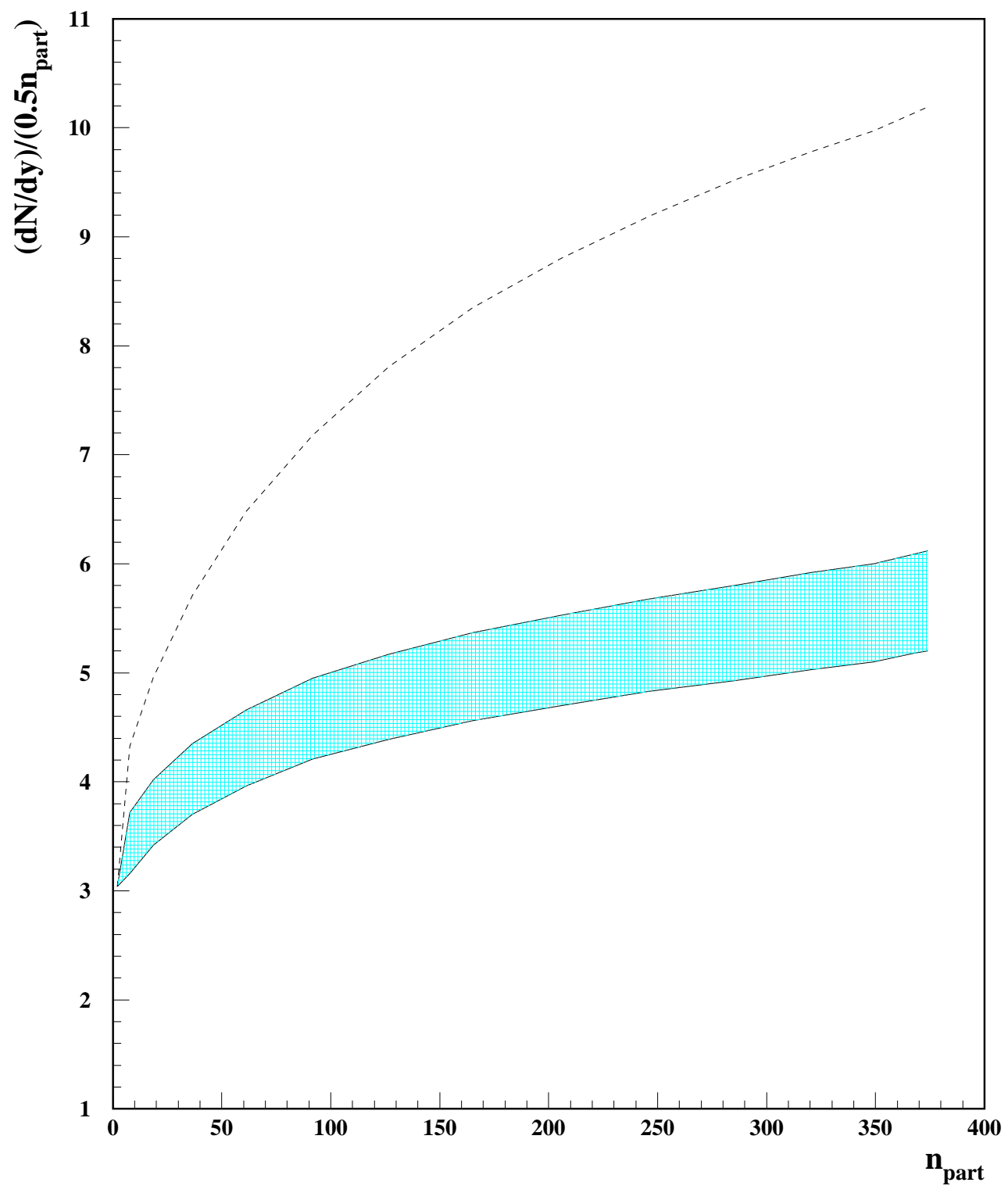


Fig. 4

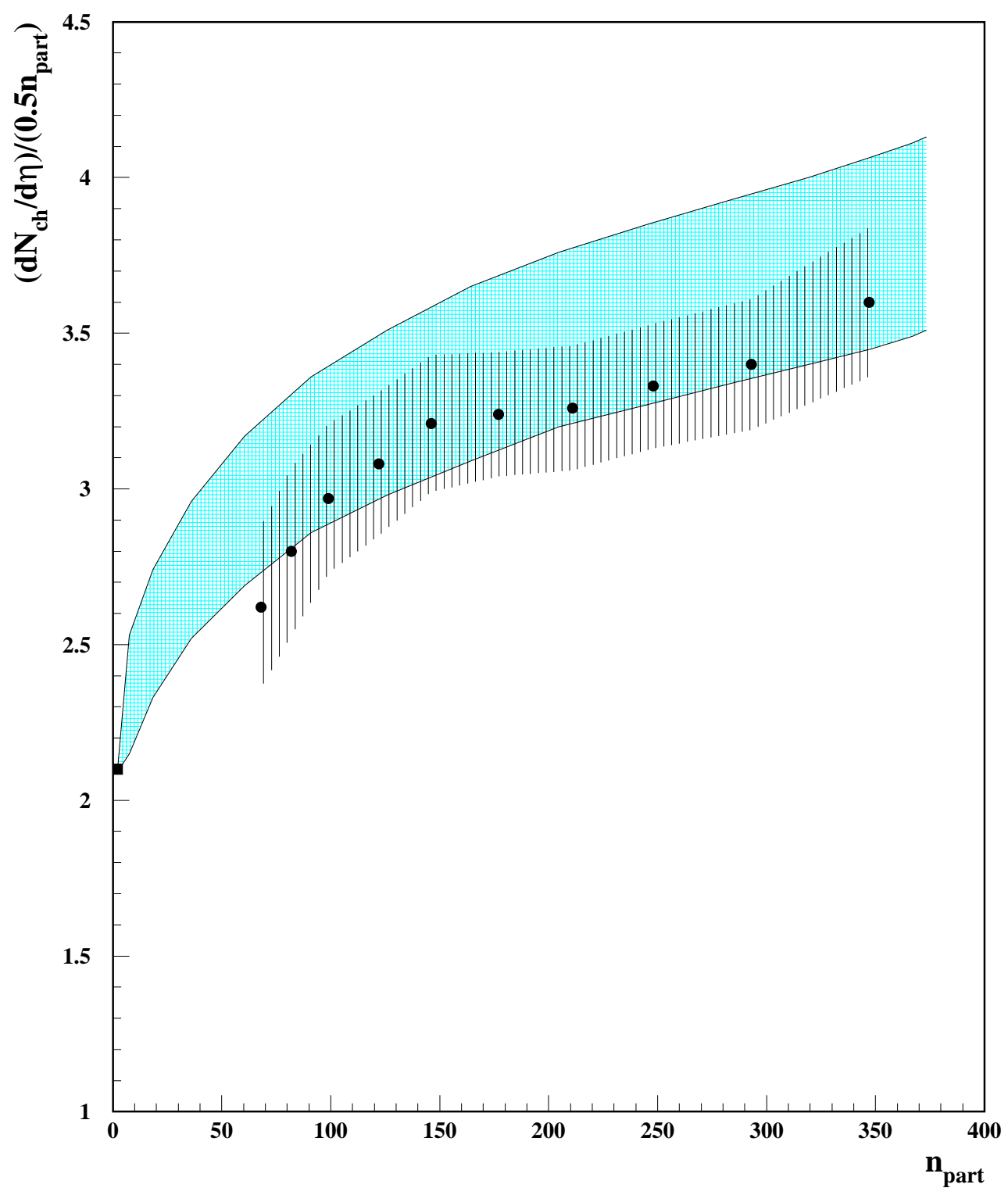




\begin{tabular}{|l|l|l|l|}
\hline$b(\mathrm{fm})$ & $N^{q q-q}$ & $N^{q-\bar{q}}$ & $R_{A u-A u}$ \\
& & & \\
\hline & & & \\
2 & 0.859 & 0.345 & 0.656 \\
4 & 0.861 & 0.347 & 0.657 \\
6 & 0.867 & 0.351 & 0.664 \\
8 & 0.875 & 0.358 & 0.681 \\
10 & 0.887 & 0.368 & 0.712 \\
12 & 0.903 & 0.381 & 0.763 \\
& & 0.397 & 0.843 \\
\hline
\end{tabular}

Table 1 